\title{
EGFR, KRAS, BRAF and ALK gene alterations in lung adenocarcinomas: patient outcome, interplay with morphology and immunophenotype
}

\author{
Arne Warth ${ }^{1,7}$, Roland Penzel ${ }^{1,7}$, Heike Lindenmaier ${ }^{1}$, Regine Brandt ${ }^{1}$, \\ Albrecht Stenzinger ${ }^{1}$, Esther Herpel ${ }^{1}$, Benjamin Goeppert ${ }^{1}$, Michael Thomas $^{2,3}$, \\ Felix J.F. Herth ${ }^{3,4}$, Hendrik Dienemann ${ }^{3,5}$, Philipp A. Schnabel ${ }^{1,3}$, \\ Peter Schirmacher ${ }^{1}$, Hans Hoffmann ${ }^{4}$, Thomas Muley ${ }^{3,6,7}$ and Wilko Weichert ${ }^{1,7}$
}

Affiliations: ${ }^{1}$ Institute of Pathology, University Hospital Heidelberg, Heidelberg, ${ }^{2}$ Dept of Thoracic Oncology, Thoraxklinik at Heidelberg University, Heidelberg. ${ }^{3}$ Translational Research Centre Heidelberg (TLRC-H), German Centre for Lung Research (DZL), Heidelberg, "Dept of Pneumology and Critical Care Medicine, Thoraxklinik at Heidelberg University, Heidelberg, ${ }^{5}$ Dept of Thoracic Surgery, Thoraxklinik at Heidelberg University, Heidelberg, and ${ }^{6}$ Translational Research Unit, Thoraxklinik at Heidelberg University, Heidelberg, Germany. ${ }^{7}$ These authors contributed equally.

Correspondence: A. Warth, Institute of Pathology, Heidelberg University, Im Neuenheimer Feld 224, D-69120 Heidelberg, Germany. E-mail: arne.warthamed.uni-heidelberg.de

ABSTRACT Numerous studies have been published on single aspects of pulmonary adenocarcinoma (ADC). To comprehensively link clinically relevant ADC characteristics, we evaluated established morphological, diagnostic and predictive biomarkers in 425 resected ADCs.

Morphology was reclassified. Cytokeratin-7, thyroid transcription factor (TTF)1, napsin A, thymidylate synthase and excision repair cross-complementing rodent repair deficiency complementation group-1 expression, anaplastic lymphoma kinase rearrangements as well as epidermal growth factor receptor (EGFR), V-Ki-ras2 Kirsten rat sarcoma viral oncogene homologue (KRAS) and v-Raf murine sarcoma viral oncogene homologue B1 (BRAF) mutations were analysed. All characteristics were correlated with clinical and survival parameters.

Morphological ADC subtypes were significantly associated with smoking history and distinct patterns of diagnostic biomarkers. KRAS mutations were prevalent in male smokers, while EGFR mutations were associated with female sex, nonsmoking and lepidic as well as micropapillary growth patterns. TTF1 expression (hazard ratio (HR) for overall survival $0.61, \mathrm{p}=0.021$ ) and $B R A F$ mutations (HR for disease-free survival 2.0, $\mathrm{p}=0.046)$ were found to be morphology- and stage-independent predictors of survival in multivariate analysis. Adjuvant radio-/chemotherapy, in some instances, strongly impacted on the prognostic effect of both diagnostic and predictive biomarkers.

Our data draw a comprehensive picture of the prevalence and interplay of established histological and molecular ADC characteristics. These data will help to develop time- and cost-effective diagnostic and treatment algorithms for ADC.

@ERSpublications

Morphological, diagnostic and predictive biomarkers, and clinical characteristics of pulmonary adenocarcinomas http://ow.ly/skRky

This article has supplementary material available from www.erj.ersjournals.com

Received: Jan 302013 | Accepted after revision: July 102013 | First published online: Aug 292013

Support statement: Parts of this study were supported by the LungSys Consortium (grant reference number 0316042B) and a research grant by Novartis.

Conflict of interest: Disclosures can be found alongside the online version of this article at www.erj.ersjournals.com

Copyright @ERS 2014 


\section{Introduction}

As the leading cause of cancer related mortality, lung cancer is a major health issue in developed countries [1]. Nonsmall cell lung cancer (NSCLC) accounts for $\sim 80 \%$ of all cases; and $\sim 60 \%$ of NSCLC are adenocarcinomas (ADCs). ADC is a complex, heterogeneous disease showing various clinicopathological and molecular characteristics with significant prognostic and predictive impact [1-10]. In particular, mutations in V-Ki-ras2 Kirsten rat sarcoma viral oncogene homologue (KRAS), epidermal growth factor receptor $(E G F R)$ and $\mathrm{v}$-Raf murine sarcoma viral oncogene homologue $\mathrm{B} 1(B R A F)$, and translocations of the anaplastic lymphoma kinase $(A L K)$ gene locus have been identified as oncogenic drivers of ADC with potential predictive value for targeted therapies. Selected patients treated with respective inhibitors have a significantly improved outcome compared with standard chemotherapy [3,5]. Furthermore, thymidylate synthase (TS) and excision repair cross-complementing rodent repair deficiency complementation group 1 protein (ERCC1) were identified as putative predictive biomarkers for pemetrexed- and platinum-based therapies, respectively [11-14]. Most recently, exome and genome sequences of ADC have been mapped, revealing novel potential therapeutic targets $[15,16]$, which, however, have mostly not yet entered clinical decision making.

The majority of ADC patients present with inoperable tumour stages at initial diagnosis. Thus, only small biopsies or cytological specimens are available for diagnostic and predictive assessment; in up to a third of all cases, diagnostic immunohistochemistry is additionally required for reliable tumour subtyping [8]. To set up effective patient stratification and reliable treatment strategies in the limited tissue setting, linkage of histomorphological, immunohistochemical, molecular and clinical data is crucial to understand the interplay between all relevant parameters.

In order to comprehensively assess established diagnostic, prognostic and predictive ADC characteristics, their associations with each other and with patient outcome, we retrospectively analysed a Caucasian cohort of 425 subsequently resected ADCs with available clinical data for histomorphology, diagnostic immunomarkers, genetic alterations of KRAS, EGFR, BRAF and $A L K$, as well as protein expression of TS and ERCC1.

\section{Patients and methods \\ Patients}

Only invasive ADCs surgically resected between 2002 and 2008 with available clinicopathological data were included. Diagnoses and subtyping were made according to the 2004 World Health Organization classification for lung cancer [17] and the novel International Association for the Study of Lung Cancer (IASLC)/American Thoracic Society (ATS)/European Respiratory Society (ERS) classification [18]. The 7th edition Union for International Cancer Control (UICC)/American Joint Committee on Cancer TNM (tumour, node, metastasis) classification was applied. Overall survival and disease-free survival (DFS) were recorded. For DFS, an event was defined as any definite clinical or pathological evidence of local or distant recurrence.

\section{Clinical characteristics}

Morphological, immunohistochemical, molecular and clinical datasets were available from 425 cases. Cases with single missing data points were not included in the specific analyses. Nine $(2.1 \%)$ patients underwent wedge resection, two (0.5\%) segmentectomy, 340 (80\%) lobectomy, $11(2.6 \%)$ bilobectomy and $63(14.8 \%)$ pneumonectomy, accompanied by systematic lymph node dissection. 264 (62.1\%) patients were male. Median (range) age at resection was 62.6 (38.3-84.8) years. $107(25.2 \%)$ patients received adjuvant chemotherapy; $73(48.7 \%)$ stage III/IV patients received adjuvant mediastinal radiotherapy. Adjuvant platinum-based chemotherapy or radiotherapy was administered, unless contraindications were present, according to the guidelines in effect at the time of resection and the clinical status of the patient. None of the patients received biomarker-based targeted therapies. Mean follow-up for patients alive at the end-point of overall survival analysis $(n=246,57.9 \%)$ was 48.2 months. Never-smokers were defined as having smoked <100 cigarettes in their lives; former and active smokers were designated as smokers. Clinicopathological characteristics of the patients included in the analyses are given in table 1.

\section{Histomorphological evaluation}

All conventional ADCs were subjected to pattern analysis according to the criteria of the IASLC/ATS/ERS classification as described previously in detail [9], recording the percentage of each histological component (lepidic, acinar, solid, papillary and micropapillary) in 5\% increments. The predominant pattern was defined as the pattern covering the largest tumour area. 


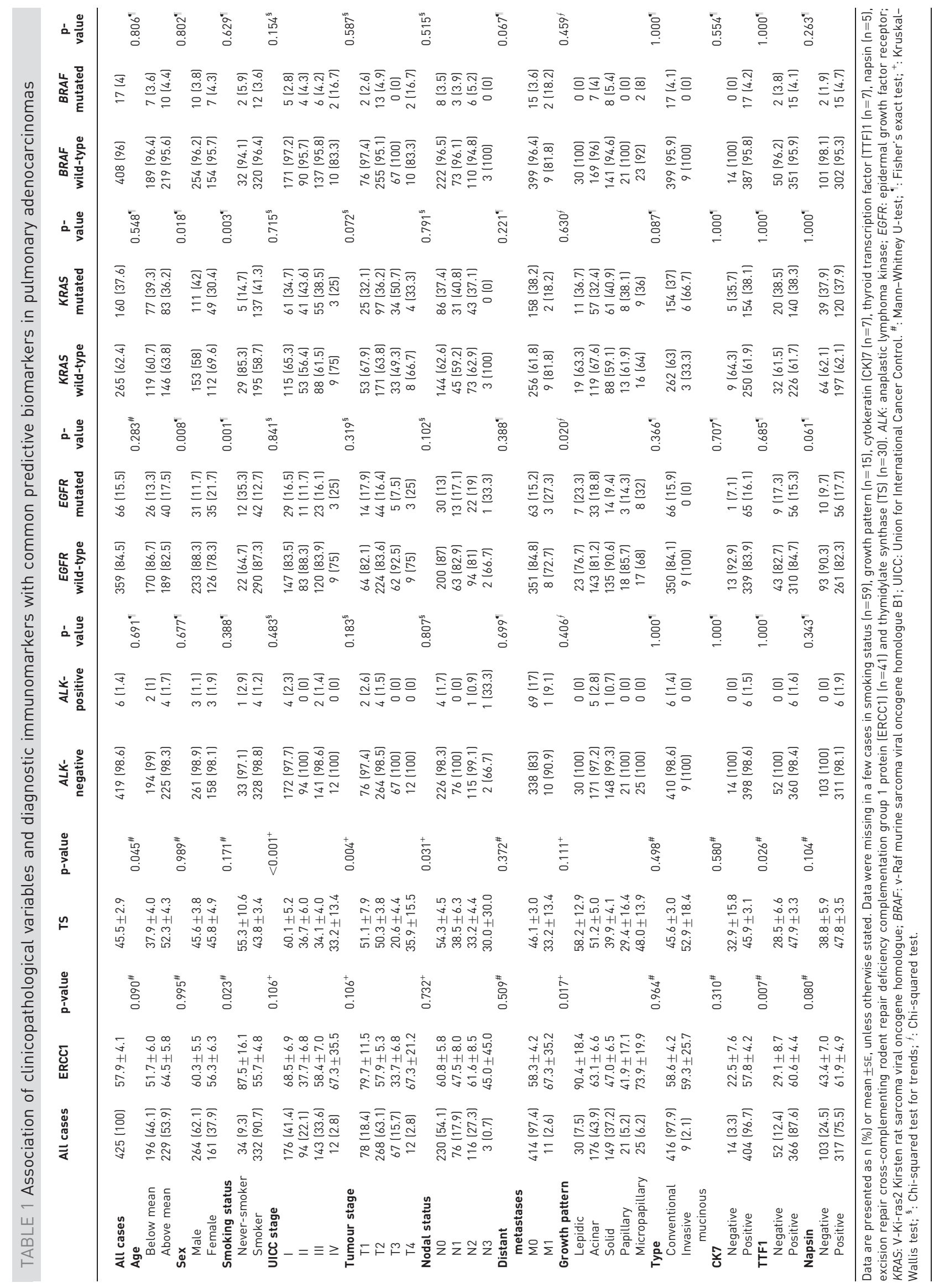


Tissue microarray construction and immunohistochemistry

For immunohistochemical analyses of diagnostic (cytokeratin (CK)7, thyroid transcription factor (TTF) 1 and napsin A) and predictive immunomarkers (TS and ERCC1), a tissue microarray (TMA) described previously in detail [8] was used. Use of all tissues was approved by the local ethics committee of the University of Heidelberg, Heidelberg, Germany (No. 206/2005). After haematoxylin- and eosin-based selection of appropriate areas, a TMA machine (ATA 27; AlphaMetrix Biotech, Rödermark, Germany) was used to extract tandem 1.0-mm cylindrical core samples from tissue donor blocks. Immunohistochemical staining was performed by the tissue bank of the National Tumour Centre (Heidelberg, Germany), using commercially available antibodies. All standard routine diagnostic antibodies were applied according to quality-controlled protocols consistently tested in round-robin trials (www.nordiqc.org) and in an accredited setting. TMA slides were deparaffinised and pretreated with an antigen retrieval buffer. Subsequent steps were carried out in a staining machine (DAKO Autostainer; Hamburg, Germany). Expression of diagnostic immunomarkers was evaluated according to a dichotomous scoring scheme [8]. Nuclear and cytoplasmic TS and ERCC1 expression were analysed separately using the $\mathrm{H}$-score $(\mathrm{H}=1 \times$ $(\%$ tumour $1+($ weak staining $))+2 \times(\%$ tumour $2+($ moderate staining $))+3 \times(\%$ tumour $3+($ strong staining $)))$. For overall assessment of TS and ERCC1 expression, the highest nuclear and/or cytoplasmic H-score for the respective marker was noted. For further details on the antibodies used see table S1.

\section{Molecular analyses}

All cases were analysed for mutations in KRAS (exon 1), EGFR (exons 18-21) and BRAF (exon 15) by Sanger sequencing. Extraction of genomic DNA was performed after manual microdissection [10] by proteinase K digestion using a fully automated purification system (QIASymphony SP; Qiagen, Hilden, Germany). For reliable sequencing analyses [10], only microdissected tissue material with $>40 \%$ tumour cell content was used. DNA content was measured using a spectrophotometer (NanoDrop; Thermo Scientific, Wilmington, DE, USA). For PCR amplification the following primers were used. EGFR: 5' -gctgaggtgaccttgtctc-3' (exon 18 forward), 5'-acagcttgcaaggactctgg-3' (exon 18 reverse); 5'-gctggtaacatccacccaga-3' (exon 19 forward), $5^{\prime}$-gagaaaaggtgggcctgag-3' (exon 19 reverse); 5' -catgtgcccctccttctg-3' (exon 20 forward), $5^{\prime}$-gatcctggctccttatctcc$3^{\prime}$ (exon 20 reverse); 5' - cagagcttcttcccatgatga-3' (exon 21 forward), 5'-cctggtgtcaggaaaatgct-3' (exon 21 reverse); KRAS: $5^{\prime}$-gtgtgacatgttctaatatagtca-3' (exon 1 forward), $5^{\prime}$-gaatggtcctgcaccagtaa- $3^{\prime}$ (exon 1 reverse); BRAF: $5^{\prime}$-cctaaactcttcataatgcttgctc-3' (exon 15 forward), 5'-ccacaaaatggatccagaca-3' (exon 15 reverse). Direct sequencing of the PCR amplicons was performed for both strands on a 3500 Genetic Analyzer using the BigDye Terminator v1.1 Cycle Sequencing Kit (both Applied Biosystems, Life Technologies, Carlsbad, CA, USA).

To identify cases with $A L K$ rearrangements, all cases were screened using immunohistochemistry using a sensitive antibody for the detection of ALK-positive NSCLC [19]. Positive cases were subjected to fluorescence in situ hybridisation (FISH) using a break-apart probe (Vysis, Abbott Laboratories, Abbott Park, IL, USA) as described previously [6]. Only cases with FISH-confirmed ALK rearrangement were considered ALK-positive.

\section{Statistics}

Correlation of categorical biomarkers with clinicopathological data was performed using Fisher's exact test, Chi-squared test and Chi-squared test for trends as indicated. Semi-quantitatively evaluated biomarkers (ERCC1 and TS) were compared with clinicopathological data using Mann-Whitney U-tests and KruskalWallis tests. Overall survival and DFS were estimated using the Kaplan-Meier method, with a log-rank test to probe for significance. Hazard ratios (HRs) for univariate and multivariate survival analyses were calculated using the Cox proportional hazard model. All statistical analyses were performed using SPSS Statistics 20 (IBM, Ehningen, Germany). p $<0.05$ was considered significant.

\section{Results}

Distribution of pathological, diagnostic and molecular biomarker characteristics in pulmonary ADC The final cohort consisted of 416 (97.9\%) conventional and nine (2.1\%) invasive mucinous ADCs. Of the conventional ADCs, 30 (7.5\%) were lepidic, 176 (43.9\%) acinar, 21 (5.2\%) papillary, 25 (6.2\%) micropapillary and $149(37.2 \%)$ solid predominant. In 15 cases, the existing archival tissue was not sufficient for a reliable morphological reclassification.

Concerning the diagnostic biomarkers, $96.7 \%, 87.6 \%$ and $75.5 \%$ of ADC cases expressed CK7, TTF1 and napsin A, respectively.

$A L K$ translocations were identified in six (1.4\%) cases. KRAS, EGFR and BRAF mutations were detected in $160(37.6 \%), 66(15.5 \%)$ and 17 cases $(4 \%)$, respectively. 
Double mutations (mutation 1/mutation 2) occurred as KRAS/KRAS ( $\mathrm{n}=8,1.9 \%), E G F R / E G F R(\mathrm{n}=6$, $1.4 \%), \operatorname{KRAS} / E G F R(\mathrm{n}=6,1.4 \%)$ and EGFR/BRAF $(\mathrm{n}=2,0.5 \%)$. One out of six ALK translocated ADCs showed an additional KRAS mutation. Mean H-scores for ERCC1 and TS were 57.9 and 45.5. For further details on the distribution of clinicopathological characteristics, see table 1.

\section{Association of selected clinical and morphological parameters}

Distribution of UICC stages and TNM classification parameters significantly differed when compared with the dominant growth pattern. Furthermore, predominant histomorphology correlated with smoking status; acinar (91.2\% of cases), solid (95.7\% of cases) and papillary (89.5\% of cases) tumours were significantly more likely to occur in smokers or ex-smokers than lepidic $(69.2 \%$ of cases) or micropapillary (81\% of cases $)$ predominant ADCs $(\mathrm{p}<0.001)$. For a comprehensive overview of further findings, compare figure 1 and table 1 .

\section{Association of diagnostic and predictive biomarkers with clinical parameters}

Apart from TTF1, whose expression was significantly more prevalent in tumours with low pathological tumour ( $\mathrm{pT})$ stages $(\mathrm{p}=0.035)$, there were no significant associations of the other diagnostic immunomarkers (CK7 and napsin A) with staging parameters (table 1).

EGFR mutations were significantly more frequent in tumours of female patients, while KRAS mutations were more frequent in males (table 1 and fig. 2). TS expression was significantly higher in tumours of older patients. ERCC1 expression was significantly higher $(\mathrm{p}=0.023)$ in tumours associated with smoking history. Of note, smoking history was also associated with higher rates of KRAS mutations $(\mathrm{p}=0.003)$ and lower rates of EGFR mutations ( $\mathrm{p}=0.001)$; the other analysed biomarkers showed no significant association with smoking (table 1).

$A L K$ translocations and EGFR, KRAS and BRAF mutations were not significantly associated with staging parameters (table 1 and fig. 2).

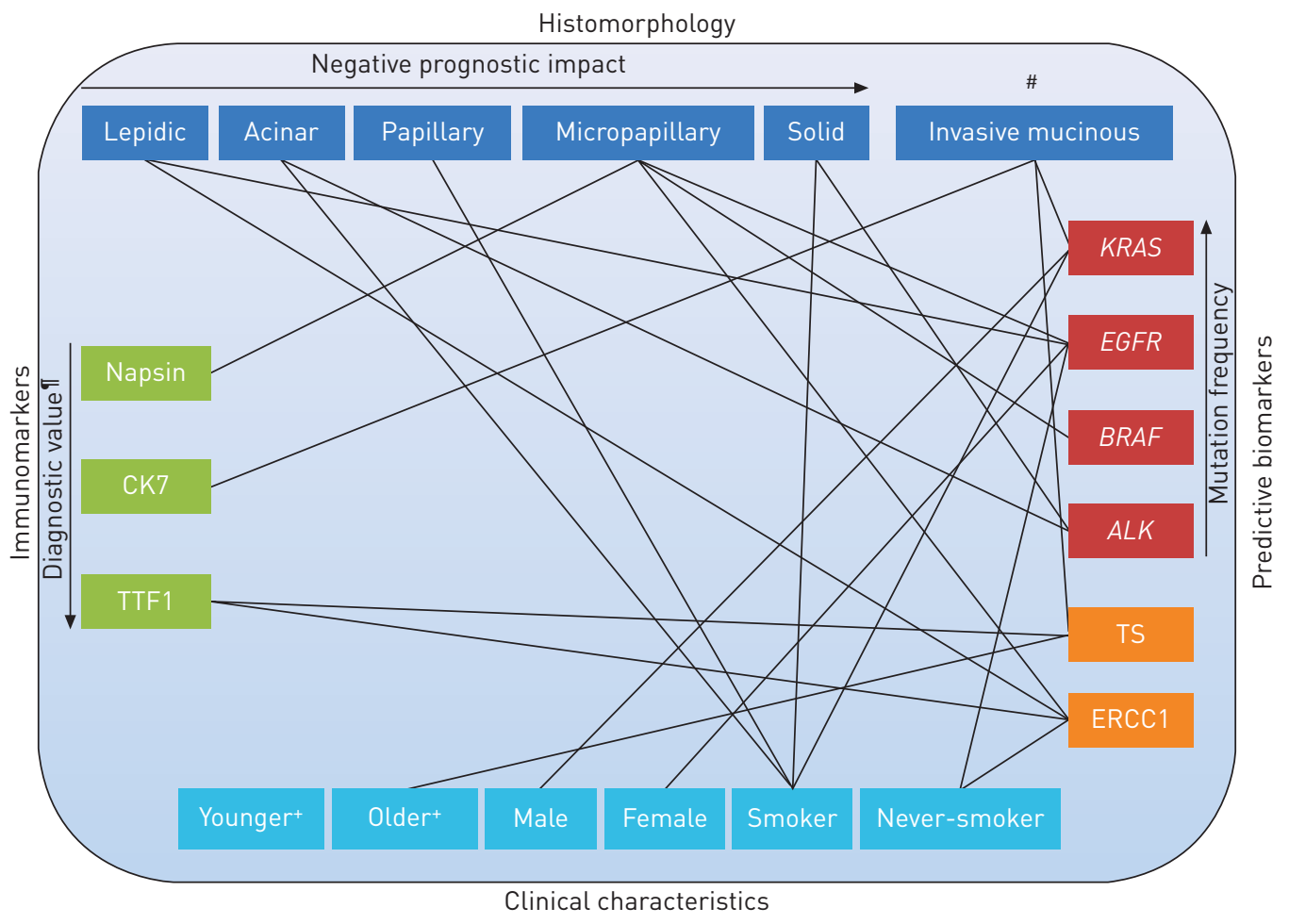

FIGURE 1 Interplay between clinicopathological variables, and diagnostic and predictive biomarkers in pulmonary adenocarcinoma. Lines indicate a positive association of the respective parameters. CK: cytokeratin; TTF: thyroid transcription factor; KRAS: V-Ki-ras2 Kirsten rat sarcoma viral oncogene homologue; EGFR: epidermal growth factor receptor; BRAF: v-Raf murine sarcoma viral oncogene homologue B1; $A L K$ : anaplastic lymphoma kinase; TS: thymidylate synthase; ERCC1: excision repair cross-complementing rodent repair deficiency complementation group 1 protein. \#: prognostic impact to be determined; ${ }^{\top}$ : compare with WARTH et al. [8]; ${ }^{+}$: patients were classified as being younger or older than the mean. 
a)

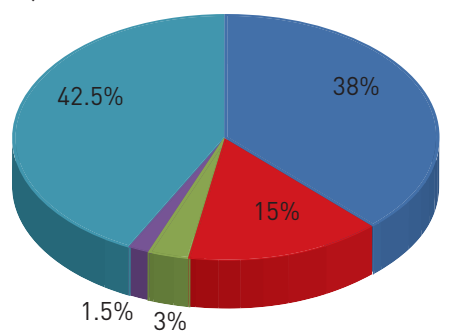

d)

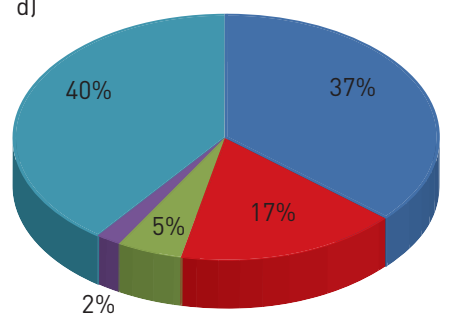

- KRAS mutated

- EGFR mutated

BRAF mutated

- ALK rearrangement

- Unknown b)

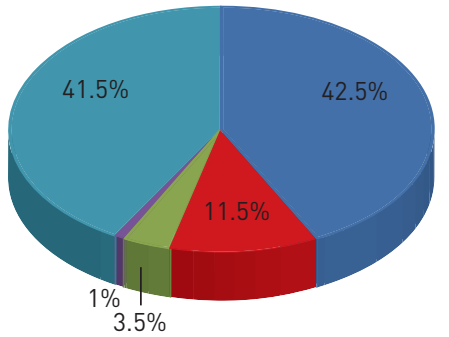

e)

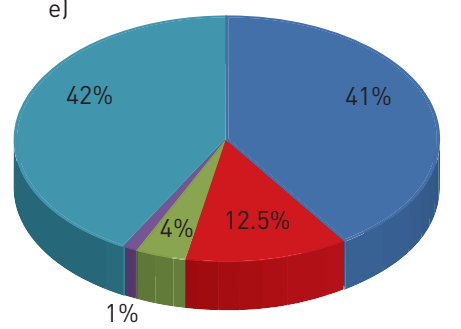

c)
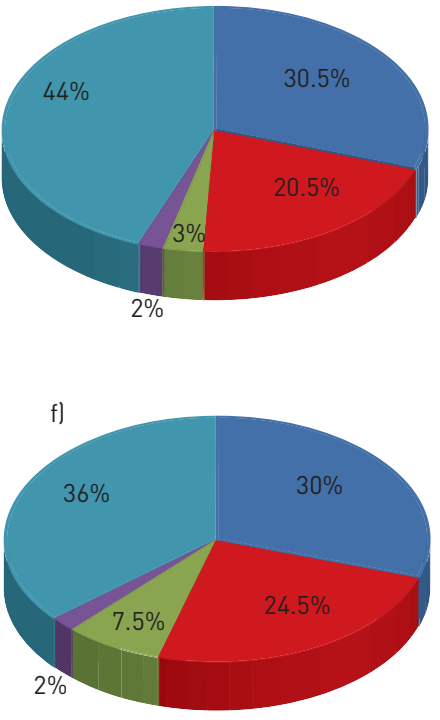

FIGURE 2 Distributions of common oncogenic driver mutations according to stage and sex in 425 pulmonary adenocarcinomas. a) Stage I-II total $(n=275)$; b) stage I-II male $(n=167)$; c) stage I-II female $(n=108)$; d) stage III-IV total $(n=150)$; e) stage III-IV male $(n=97)$; stage III-IV female $(n=53)$. KRAS: V-Ki-ras2 Kirsten rat sarcoma viral oncogene homologue; EGFR: epidermal growth factor receptor; BRAF: v-Raf murine sarcoma viral oncogene homologue B1; ALK: anaplastic lymphoma kinase.

\section{Association of diagnostic and predictive biomarkers with morphological characteristics}

Napsin expression was significantly associated with predominant histomorphological pattern (fig. S1) $(\mathrm{p}<0.001)$, with micropapillary carcinomas showing the highest expression rate, while solid and lepidic carcinomas were more likely to be negative. KRAS mutations were more frequent in invasive mucinous ADCs, while no other types of the analysed driver mutations were found in this ADC subtype (table 1). Of all analysed molecular alterations, only EGFR mutation frequency was significantly different with respect to growth pattern, with lepidic and micropapillary predominant ADCs showing higher EGFR mutation rates (table 1), mainly due to differences in the frequency of exon 19 mutations (fig. S2). ALK translocations were exclusively seen in acinar and solid predominant ADCs (fig. S2) and BRAF mutations were predominantly found in micropapillary but not in papillary or lepidic predominant ADCs (table 1 and fig. 1).

\section{Association of diagnostic and predictive biomarkers with each other}

Both TS and ERCC1 expression were significantly higher in TTF1-positive ADCs $(p=0.026$ and $p=0.007$, respectively) (table 1). Furthermore, higher expression levels of ERCC1 and TS were associated with ALK translocations and wild-type KRAS (ERCC1 $\mathrm{p}=0.005$ and 0.008 , respectively, and TS $\mathrm{p}=0.075$ and 0.049 , respectively) (fig. S3).

Prognostic value of diagnostic and predictive biomarkers in pulmonary ADC

TTF1 and napsin A expression were associated with prolonged survival with a stronger prognostic value for TTF1 (table 2). Furthermore, TTF1 expression was a stage- and pattern-independent predictor of overall survival (HR 0.61, p=0.021). The survival effect was specifically evident in patients without adjuvant chemotherapy (table S2). TS expression was a significant predictor of better patient survival for overall survival and DFS (table S3 and fig. S4). However, when survival impact of TS expression was adjusted for stage and dominant histomorphological pattern in a Cox regression model, TS failed to show independent impact on patient survival (overall survival HR $0.82, \mathrm{p}=0.233$ and DFS HR $0.73, \mathrm{p}=0.073$ ). The presence of $B R A F$ mutations was a negative prognostic factor for DFS $(\mathrm{p}=0.009)$ but not for overall survival (fig. S5). Interestingly, although overall no significant differences in survival were noted, patients with EGFR 
TABLE 2 Overall and disease-free survival in dependence of clinicopathological, diagnostic and predictive biomarkers

Overall survival

Disease-free survival

\begin{tabular}{lll}
\hline Cases $\quad$ Events $\quad$ Survival months & p-value
\end{tabular}

Cases Events Survival months p-value

\begin{tabular}{|c|c|c|c|c|c|c|c|c|}
\hline \multicolumn{9}{|l|}{ Age } \\
\hline Below mean & 196 & 80 & $65.32 \pm 3.43$ & \multirow[t]{2}{*}{0.644} & 196 & 101 & $50.67 \pm 3.46$ & \multirow[t]{2}{*}{0.006} \\
\hline Above mean & 229 & 99 & $60.91 \pm 3.17$ & & 229 & 84 & $63.12 \pm 3.52$ & \\
\hline \multicolumn{9}{|l|}{ Sex } \\
\hline Male & 264 & 123 & $59.76 \pm 2.94$ & \multirow[t]{2}{*}{0.024} & 264 & 124 & $53.00 \pm 3.06$ & \multirow[t]{2}{*}{0.045} \\
\hline Female & 161 & 56 & $69.35 \pm 3.77$ & & 161 & 61 & $64.06 \pm 4.12$ & \\
\hline \multicolumn{9}{|l|}{ Smoking status } \\
\hline Never-smoker & 34 & 8 & $57.05 \pm 3.44$ & \multirow[t]{2}{*}{0.068} & 34 & 15 & $41.39 \pm 5.03$ & \multirow[t]{2}{*}{0.959} \\
\hline Smoker & 332 & 144 & $62.23 \pm 2.68$ & & 332 & 148 & $55.04 \pm 2.80$ & \\
\hline \multicolumn{9}{|l|}{ UICC stage } \\
\hline I & 176 & 41 & $83.07 \pm 3.17$ & \multirow{4}{*}{$<0.001$} & 176 & 42 & $79.18 \pm 3.28$ & \multirow{4}{*}{$<0.001$} \\
\hline II & 94 & 43 & $61.05 \pm 4.60$ & & 94 & 51 & $47.99 \pm 4.89$ & \\
\hline III & 143 & 87 & $41.65 \pm 3.68$ & & 143 & 85 & $34.70 \pm 3.83$ & \\
\hline IV & 12 & 8 & $20.25 \pm 4.06$ & & 12 & 7 & $16.10 \pm 5.20$ & \\
\hline \multicolumn{9}{|l|}{ Tumour stage } \\
\hline T1 & 78 & 15 & $82.12 \pm 4.92$ & \multirow{4}{*}{$<0.001$} & 78 & 17 & $77.23 \pm 5.35$ & \multirow{4}{*}{$<0.001$} \\
\hline $\mathrm{T} 2$ & 268 & 122 & $61.91 \pm 2.85$ & & 268 & 120 & $56.95 \pm 3.04$ & \\
\hline T3 & 67 & 34 & $54.69 \pm 5.83$ & & 67 & 41 & $37.47 \pm 5.70$ & \\
\hline T4 & 12 & 8 & $37.32 \pm 11.14$ & & 12 & 7 & $17.50 \pm 4.14$ & \\
\hline Nodal status & & & & & & & & \\
\hline NO & 230 & 63 & $79.27 \pm 2.89$ & & 230 & 74 & $70.70 \pm 3.11$ & \\
\hline N1 & 76 & 44 & $49.44 \pm 5.04$ & $<0.001$ & 76 & 41 & $45.17 \pm 5.84$ & $<0.001$ \\
\hline N2 & 116 & 71 & $40.36 \pm 4.03$ & & 116 & 69 & $31.66 \pm 3.51$ & \\
\hline N3 & 3 & 1 & $13.13 \pm 0.00$ & & 3 & 1 & $8.82 \pm 1.87$ & \\
\hline Distant metastases & & & & & & & & \\
\hline MO & 413 & 171 & $64.42 \pm 2.37$ & $<0.001$ & 413 & 178 & $58.12 \pm 2.52$ & 0.006 \\
\hline M1 & 12 & 8 & $20.25 \pm 4.06$ & & 12 & 7 & $16.10 \pm 5.20$ & \\
\hline Pattern & & & & & & & & \\
\hline Lepidic & 30 & 8 & $70.21 \pm 6.60$ & & 30 & 10 & $65.74 \pm 7.00$ & \\
\hline Acinar & 176 & 72 & $65.96 \pm 3.52$ & 0.071 & 176 & 72 & $60.92 \pm 3.78$ & 0.051 \\
\hline Solid & 149 & 72 & $56.36 \pm 3.83$ & & 149 & 71 & $50.68 \pm 4.22$ & \\
\hline Papillary & 21 & 10 & $46.16 \pm 7.45$ & & 21 & 12 & $35.12 \pm 6.63$ & \\
\hline Micropapillary & 25 & 11 & $47.19 \pm 6.96$ & & 25 & 13 & $38.20 \pm 7.29$ & \\
\hline Type & & & & & & & & \\
\hline Conventional & 416 & 178 & $62.64 \pm 2.37$ & 0.046 & 416 & 185 & $56.11 \pm 2.53$ & 0.009 \\
\hline Invasive mucinous & 9 & 1 & $87.04 \pm 8.51$ & & 9 & 0 & & \\
\hline CK7 & & & & & & & & \\
\hline Negative & 14 & 5 & $48.66 \pm 5.85$ & 0.569 & 14 & 7 & $35.45 \pm 7.14$ & 0.701 \\
\hline Positive & 404 & 171 & $63.27 \pm 2.40$ & & 404 & 177 & $57.10 \pm 2.55$ & \\
\hline TTF1 & & & & & & & & \\
\hline Negative & 52 & 26 & $49.80 \pm 61.09$ & 0.030 & 52 & 26 & $43.61 \pm 6.51$ & 0.045 \\
\hline Positive & 366 & 150 & $64.75 \pm 24.99$ & & 366 & 157 & $58.50 \pm 2.67$ & \\
\hline Napsin & & & & & & & & \\
\hline Negative & 103 & 48 & $58.04 \pm 4.68$ & 0.160 & 103 & 51 & $50.67 \pm 4.96$ & 0.056 \\
\hline Positive & 317 & 129 & $64.84 \pm 2.69$ & & 317 & 133 & $58.97 \pm 2.89$ & \\
\hline ERCC1 & & & & & & & & \\
\hline Below mean & 198 & 84 & $62.24 \pm 3.51$ & 0.509 & 198 & 94 & $52.30 \pm 3.62$ & 0.072 \\
\hline Above mean & 186 & 79 & $63.98 \pm 3.40$ & & 186 & 78 & $59.44 \pm 3.75$ & \\
\hline TS & & & & & & & & \\
\hline Below mean & 208 & 95 & $56.35 \pm 3.31$ & 0.005 & 208 & 102 & $49.29 \pm 3.53$ & $<0.001$ \\
\hline Above mean & 187 & 66 & $71.84 \pm 3.43$ & & 187 & 66 & $65.85 \pm 3.88$ & \\
\hline$A L K$ & & & & & & & & \\
\hline Negative & 419 & 178 & $63.30 \pm 2.35$ & 0.387 & 419 & 183 & $57.41 \pm 2.52$ & 0.615 \\
\hline Positive & 6 & 1 & $47.88 \pm 7.84$ & & 6 & 2 & $50.05 \pm 4.22$ & \\
\hline KRAS & & & & & & & & \\
\hline Wild-type & 265 & 111 & $64.28 \pm 2.90$ & 0.464 & 265 & 114 & $58.96 \pm 3.10$ & 0.302 \\
\hline Mutant & 160 & 68 & $59.76 \pm 3.55$ & & 160 & 71 & $52.24 \pm 3.94$ & \\
\hline BRAF & & & & & & & & \\
\hline Wild-type & 408 & 170 & $63.81 \pm 2.40$ & 0.398 & 408 & 172 & $59.19 \pm 2.55$ & 0.009 \\
\hline Mutant & 17 & 9 & $55.13 \pm 10.92$ & & 17 & 13 & $29.47 \pm 6.16$ & \\
\hline
\end{tabular}




\begin{tabular}{|c|c|c|c|c|c|c|c|c|}
\hline & \multicolumn{4}{|c|}{ Overall survival } & \multicolumn{4}{|c|}{ Disease-free survival } \\
\hline & Cases & Events & Survival months & p-value & Cases & Events & Survival months & $\mathrm{p}$-value \\
\hline \multicolumn{9}{|l|}{ EGFR total } \\
\hline Wild-type & 359 & 150 & $63.85 \pm 2.55$ & 0.777 & 359 & 154 & $57.78 \pm 2.71$ & 0.529 \\
\hline Mutant & 66 & 29 & $60.38 \pm 5.72$ & & 66 & 31 & $54.92 \pm 6.46$ & \\
\hline \multicolumn{9}{|c|}{ EGFR exon 18} \\
\hline \multicolumn{9}{|l|}{ EGFR exon 19} \\
\hline Wild-type & 398 & 169 & $63.16 \pm 2.43$ & 0.543 & 398 & 170 & $58.19 \pm 2.59$ & 0.214 \\
\hline Mutant & 27 & 10 & $67.82 \pm 8.73$ & & 27 & 15 & $46.96 \pm 9.07$ & \\
\hline \multicolumn{9}{|c|}{ EGFR exon 20} \\
\hline Wild-type & 413 & 172 & $63.88 \pm 2.38$ & 0.317 & 413 & 179 & $57.48 \pm 2.54$ & 0.626 \\
\hline Mutant & 12 & 7 & $52.41 \pm 11.95$ & & 12 & 6 & $55.90 \pm 13.95$ & \\
\hline
\end{tabular}

Data are presented as $\mathrm{n}$ or mean $\pm \mathrm{SE}$, unless otherwise stated. $\mathrm{p}$-values were calculated using a log-rank test. UICC: Union for International Cancer Control; CK: cytokeratin; TTF: thyroid transcription factor; ERCC1: excision repair cross-complementing rodent repair deficiency complementation group 1 protein; TS: thymidylate synthase; ALK: anaplastic lymphoma kinase; KRAS: V-Ki-ras2 Kirsten rat sarcoma viral oncogene homologue; BRAF: v-Raf murine sarcoma viral oncogene homologue B1; EGFR: epidermal growth factor receptor.

mutations receiving adjuvant chemotherapy clearly showed an improved outcome compared to patients without such therapy (table S3). A comprehensive overview of the strength of prognostic associations of all morphological, clinical and molecular biomarkers is given in table 2 and in figure 3.

\section{Discussion}

Although a wealth of details on clinical, morphological and molecular biomarkers in pulmonary ADC has been published, comprehensive studies covering all clinical and pathological characteristics relevant for current routine diagnostic setting are so far lacking. Here, we demonstrate that several specific clinical, histomorphological, immunohistochemical and molecular parameters are tightly linked or occur almost
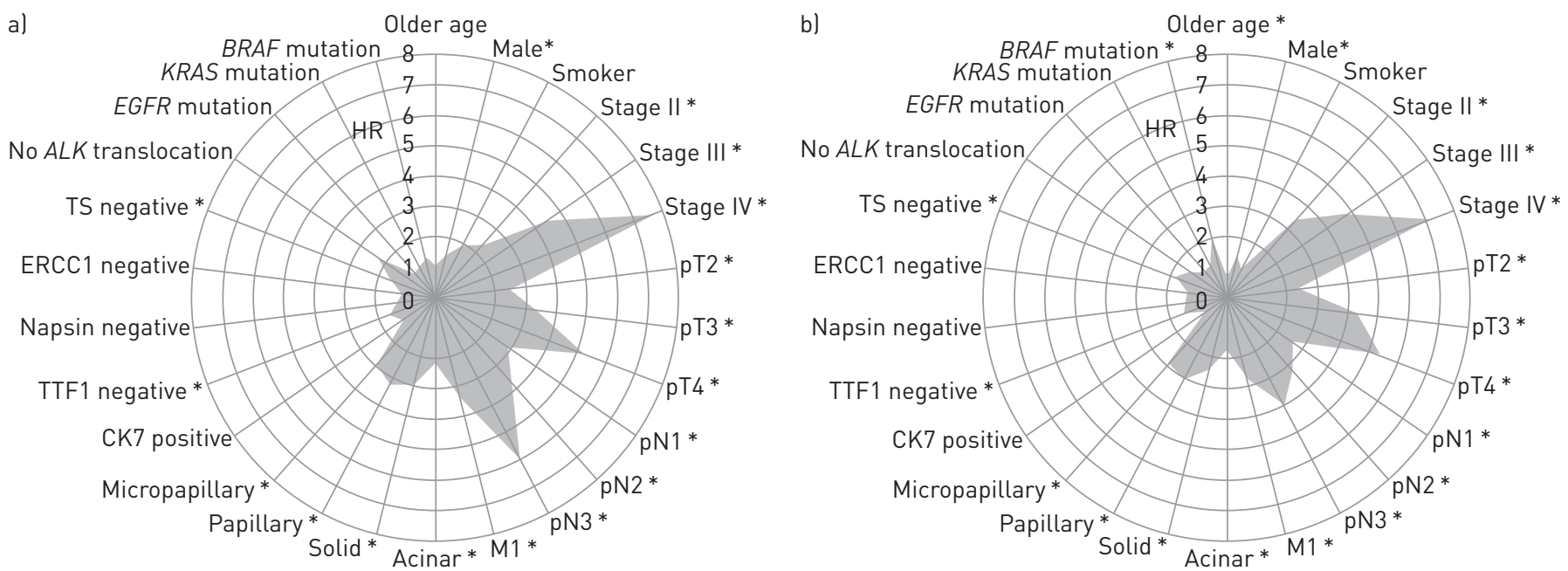

FIGURE 3 Spider web diagrams depicting the hazard ratios (HR) from univariate survival analysis of the analysed parameters including older age (HR reference:

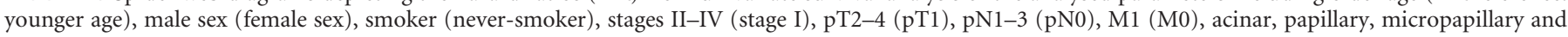
solid predominant pattern (lepidic predominant), cytokeratin (CK)7-positive (CK7-negative), thyroid transcription factor (TTF)1-negative (TTF1-positive), napsin-negative (napsin-positive), excision repair cross-complementing rodent repair deficiency complementation group 1 protein (ERCC)1-negative (ERCC1positive), thymidylate synthase (TS)-negative (TS-positive), no anaplastic lymphoma kinase (ALK) translocation ( $A L K$ translocation), epidermal growth factor receptor $(E G F R)$ mutation (EGFR wild-type), V-Ki-ras2 Kirsten rat sarcoma viral oncogene homologue (KRAS) mutation (KRAS wild-type) and v-Raf murine sarcoma viral oncogene homologue $\mathrm{B} 1(B R A F)$ mutation $\left(B R A F\right.$ wild-type) for a) overall and b) disease-free survival. ${ }^{*}$ : $\mathrm{p}<0.05$, statistically significant. 
mutually exclusively, which may have significant impact on the development of rational, tissue-sparing diagnostic algorithms as well as an optimised patient stratification.

The past decade in lung cancer research was dominated by large-scale molecular approaches to identify prognostic and predictive markers for personalised medicine. Up to now, however, only EGFR mutations and $A L K$ translocations have been successfully translated into the diagnostic setting; several other potential biomarkers have failed to achieve this goal. Molecular characterisation and subsequent clinical trials underlined that morphological features (e.g. squamous versus nonsquamous) are crucial for therapy selection. Today, it is known that the group of pulmonary ADCs is more heterogeneous than expected, with diverse biological behaviour and prognosis. Therefore, reclassification of ADCs based on histomorphology [18] was a logical and essential step with highly significant prognostic and, probably, predictive value [9]. With this novel and largely reproducible [20,21] tool, linkage of the different ADC histotypes to diagnostic and predictive biomarkers as well as clinical characteristics is essential for a comprehensive interdisciplinary classification of ADC in the future [18]. Furthermore, different therapeutic targets were found to be associated with each other, implying combined inhibitory strategies for optimised treatment algorithms. For example, ALK translocations were found to be associated with TS expression [22], EGFR mutations were reported more frequently in ERCC1-negative tumours [23] and EGFR inhibitors are known to downregulate TS $[24,25]$. Moreover, novel agents like lapatinib, a dual EGFR and Her2 tyrosine kinase inhibitor (TKI), crizotinib (ALK/c-Met inhibitor) or sorafenib (multikinase inhibitor) are directed against more than one target and may thus successfully prevent tumour escape mechanisms. Acquired resistance to targeted therapies is also closely linked to specific molecular alterations [26]. Hence, combined treatment approaches require correlative prevalence data of the respective predictive biomarkers.

ERCC1 and TS are involved in DNA synthesis and repair and their loss of expression was considered as predictive for response to platinum-based [27] and pemetrexed-based [28] chemotherapies, respectively. However, in addition to the potentially negative predictive value of both proteins, high expression levels have also been reported to be associated with an improved outcome [27, 29-31], which was confirmed, at least for TS, by this study. This is explained by the hypothesis that by preventing mutagenesis, DNA repair not only prevents cancer but also inhibits molecular events related to tumour progression. Thus, high expression of the respective markers may indicate an improved outcome in untreated patients by identifying tumours that have only slowly progressed at the molecular level [32], which is also reflected by our finding that high TS and ERCC1 expression levels were more prevalent in early tumour stages (table 1). The association of TS expression with invasive mucinous ADCs, TTF1 positivity, older age and ALK translocations [22], but also the resulting TS downregulation by TKI [24, 25] might be used for the stratification of patients towards combined therapies with pemetrexed.

Several studies have reported the predictive value of ERCC1 for platinum-based chemotherapy, including the International Adjuvant Lung Cancer Trial (IALT) on 761 NSCLC tumours [11]. However, subsequent studies indicate that this association might be specifically prominent in squamous cell carcinoma but not in ADC [33] (A. Warth, personal communication). In our ADC cohort, ERCC1 expression was also associated with reduced survival in those patients receiving adjuvant platinum-based chemotherapy; however, these differences failed to reach statistical significance, which might indicate a comparably small predictive value of this marker but, conversely, might also be attributed to the relatively small sample size in this subcohort. Most recently, FRIBOULET et al. [34] reported that they were not able to validate the predictive effect of immunostaining for ERCC1 in several large NSCLC cohorts, including the cohort from the IALT trial, which might be due to the fact that commercially available antibodies, including the one used in this study, do not seem to specifically detect the unique functional ERCC1 isoform. Thus, the suggested predictive value of ERCC1 might be disputed until more specific antibodies allow for a validation of the predictive effect of ERCC1 expression.

EGFR mutations have been reported to accumulate in young, female, Asian and never-smoking patients. However, most of the studies published to date reported on selected cohorts in the context of clinical trials. In the herein-analysed unselected Caucasian cohort, we could confirm the predominance of female sex and, in addition, found a higher prevalence in lepidic and micropapillary predominant ADCs. Age was not associated with differences in the prevalence of EGFR mutations. In contrast, others found a higher prevalence of EGFR mutations in older patients and an association to acinar-predominant ADCs [35]. These differences might be explained by different ethnic backgrounds of the respective cohorts. In any case, morphological criteria seem to be helpful if a pre-selection of patients for EGFR mutation testing is desired.

Although no specific targeted therapies exist so far, KRAS mutations are perceived as a potential negative predictive factor for TKI-based ADC treatment and a prognostic factor for surgically resected early-stage ADC [36, 37]. However, there is also evidence that KRAS mutations, which are more prevalent in males, smokers and invasive mucinous ADCs [15, 35, 38] (fig. 2), are not an a priori negative factor for TKI 
administration [39]. Agents targeting downstream effectors of the KRAS pathway may provide treatment options for this large ADC subgroup. Patient stratification for KRAS mutation testing could be performed based on the above outlined characteristics.

In first trials, BRAF inhibitors showed apparent antitumour activity in NSCLC [40] and specific BRAF mutations also rendered tumours responsive to dasatinib [41]. BRAF mutations were reported to be associated with female sex and smoking. Furthermore, BRAF-mutated tumours have been suggested to belong to an aggressive histotype, characterised by micropapillary features and shorter DFS and overall survival $[42,43]$. We could confirm an association of BRAF mutations with micropapillary-predominant $A D C$ and a significant correlation of BRAF mutations with worse DFS, but not the proposed association to female sex or smoking.

Among the diagnostic markers, TTF1 was most recently reported as an independent predictor of survival [44], which was confirmed by our study. Others also described an independent prognostic effect for napsin $\mathrm{A}$ and an association of napsin A expression with the presence of EGFR mutations in an Asian ADC cohort [45]. However, we could only see borderline associations of napsin A with survival parameters and no significant association with EGFR mutations. Again, these differing findings might be attributed to differences with respect to ethnic background and underlines the need for reliable prevalence data of large ADC cohorts from various geographical regions.

One limitation of this study is its retrospective nature. The assessment of multiple clinicopathological characteristics necessarily results in small groups for comparison and thus hampers multivariate analyses in some instances. Furthermore, predictive biomarkers are usually not analysed using resection specimens but in advanced tumour stages or recurrent tumours where only sparse tissue is available. Although TMA-based assessment of immunomarkers may largely reflect the biopsy constellation, we cannot exclude a slight prevalence shift of the molecular alterations compared to the daily routine setting.

Considering the growing number of clinically relevant biomarkers, the establishment of not only tissuesparing diagnostic algorithms but also the development of time- and cost-effective multitesting platforms for molecular alterations with respective implementation into routine diagnostics seems to be mandatory in order to fulfil the requirements of evidence-based decision making within the context of personalised treatment of lung cancer. Novel subgenomic massive parallel sequencing (MPS) strategies, which allow for a comprehensive mutational screen of tumour material in just one sequencing run are especially promising in this regard. However, prior to widespread routine diagnostic use, each of the MPS technologies applied must be adapted to the specific needs in lung cancer diagnosis; specifically, the robustness of the respective methods must be shown on small bronchial biopsy samples and paraffin embedded material.

Taken together, this is the first large-scale study covering in parallel established morphological, diagnostic and predictive biomarkers as well as clinical characteristics of pulmonary ADC. The herein presented data of a largely unselected Caucasian cohort not treated with biomarker-based targeted therapies may form a basis for the development of rational diagnostic stratification algorithms for the selection of appropriate therapies and may also serve as a source of prevalence data for the design of clinical trials.

\section{Acknowledgements}

J. Schmitt, A. Brüntgens, C. Hofherr, W. Schmitz, M. Karipidis, M. Viole (Institute for Pathology, Heidelberg, Germany) and C. Stolp (Thoraxklinik Heidelberg, Heidelberg, Germany) are acknowledged for their excellent support of this study.

\section{References}

Siegel R, Naishadham D, Jemal A. Cancer statistics, 2012. CA Cancer J Clin 2012; 62: 10-29.

Herpel E, Schnabel PA, Steins M, et al. Assessment of thymidylate synthase expression in biopsy specimens and corresponding resection specimens of non-small-cell lung cancer. Histopathology 2012; 61: 465-472.

3 Kwak EL, Bang YJ, Camidge DR, et al. Anaplastic lymphoma kinase inhibition in non-small-cell lung cancer. N Engl J Med 2010; 363: 1693-1703.

4 Lederlin M, Puderbach M, Muley T, et al. Correlation of radio- and histomorphological pattern of pulmonary adenocarcinoma. Eur Respir J 2013; 41: 943-951.

5 Mok TS, Wu YL, Thongprasert S, et al. Gefitinib or carboplatin-paclitaxel in pulmonary adenocarcinoma. $N$ Engl $J$ Med 2009; 361: 947-957.

6 Penzel R, Schirmacher P, Warth A. A novel EML4-ALK variant: exon 6 of EML4 fused to exon 19 of ALK. J Thorac Oncol 2012; 7: 1198-1199.

7 Warth A, Macher-Goeppinger S, Muley T, et al. Clonality of multifocal nonsmall cell lung cancer: implications for staging and therapy. Eur Respir J 2012; 39: 1437-1442.

8 Warth A, Muley T, Herpel E, et al. Large-scale comparative analyses of immunomarkers for diagnostic subtyping of non-small-cell lung cancer biopsies. Histopathology 2012; 61: 1017-1025.

9 Warth A, Muley T, Meister M, et al. The novel histologic International Association for the Study of Lung Cancer/ American Thoracic Society/European Respiratory Society classification system of lung adenocarcinoma is a stageindependent predictor of survival. J Clin Oncol 2012; 30: 1438-1446. 
10 Warth A, Penzel R, Brandt R, et al. Optimized algorithm for Sanger sequencing-based EGFR mutation analyses in NSCLC biopsies. Virchows Arch 2012; 460: 407-414.

11 Olaussen KA, Dunant A, Fouret P, et al. DNA repair by ERCC1 in non-small-cell lung cancer and cisplatin-based adjuvant chemotherapy. N Engl J Med 2006; 355: 983-991.

12 Olaussen KA, Mountzios G, Soria JC. ERCC1 as a risk stratifier in platinum-based chemotherapy for nonsmall-cell lung cancer. Curr Opin Pulm Med 2007; 13: 284-289.

13 Igawa S, Ryuge S, Wada M, et al. Pemetrexed for previously treated patients with non-small cell lung cancer and differences in efficacy according to thymidylate synthase expression. Chemotherapy 2012; 58: 313-320.

14 Sun JM, Han J, Ahn JS, et al. Significance of thymidylate synthase and thyroid transcription factor 1 expression in patients with nonsquamous non-small cell lung cancer treated with pemetrexed-based chemotherapy. $J$ Thorac Oncol 2011; 6: 1392-1399.

15 Govindan R, Ding L, Griffith M, et al. Genomic landscape of non-small cell lung cancer in smokers and neversmokers. Cell 2012; 150: 1121-1134.

16 Imielinski M, Berger AH, Hammerman PS, et al. Mapping the hallmarks of lung adenocarcinoma with massively parallel sequencing. Cell 2012; 150: 1107-1120.

17 Travis WD, Brambilla E, Müller-Hermelink HK, et al. eds, Pathology and Genetics of Tumours of the Lung, Pleura, Thymus and Heart. Lyon, IARC Press 2004.

18 Travis WD, Brambilla E, Noguchi M, et al. International Association for the Study of Lung Cancer/American Thoracic Society/European Respiratory Society international multidisciplinary classification of lung adenocarcinoma. J Thorac Oncol 2011; 6: 244-285.

19 Mino-Kenudson M, Chirieac LR, Law K, et al. A novel, highly sensitive antibody allows for the routine detection of ALK-rearranged lung adenocarcinomas by standard immunohistochemistry. Clin Cancer Res 2010; 16: 1561-1571.

20 Warth A, Cortis J, Fink L, et al. Training increases concordance in classifying pulmonary adenocarcinomas according to the novel IASLC/ATS/ERS classification. Virchows Arch 2012; 461: 185-193.

21 Warth A, Stenzinger A, von Brünneck AC, et al. Interobserver variability in the application of the novel IASLC/ ATS/ERS classification for pulmonary adenocarcinomas. Eur Respir J 2012; 40: 1221-1227.

22 Lee JO, Kim TM, Lee SH, et al. Anaplastic lymphoma kinase translocation: a predictive biomarker of pemetrexed in patients with non-small cell lung cancer. J Thorac Oncol 2011; 6: 1474-1480.

23 Lee KH, Min HS, Han SW, et al. ERCC1 expression by immunohistochemistry and EGFR mutations in resected non-small cell lung cancer. Lung Cancer 2008; 60: 401-407.

24 Okabe T, Okamoto I, Tsukioka S, et al. Synergistic antitumor effect of S-1 and the epidermal growth factor receptor inhibitor gefitinib in non-small cell lung cancer cell lines: role of gefitinib-induced down-regulation of thymidylate synthase. Mol Cancer Ther 2008; 7: 599-606.

25 Giovannetti E, Lemos C, Tekle C, et al. Molecular mechanisms underlying the synergistic interaction of erlotinib, an epidermal growth factor receptor tyrosine kinase inhibitor, with the multitargeted antifolate pemetrexed in nonsmall-cell lung cancer cells. Mol Pharmacol 2008; 73: 1290-1300.

26 Ohashi K, Sequist LV, Arcila ME, et al. Lung cancers with acquired resistance to EGFR inhibitors occasionally harbor BRAF gene mutations but lack mutations in KRAS, NRAS, or MEK1. Proc Natl Acad Sci USA 2012; 109: E2127-E2133.

27 Jiang J, Liang X, Zhou X, et al. ERCC1 expression as a prognostic and predictive factor in patients with non-small cell lung cancer: a meta-analysis. Mol Biol Rep 2012; 39: 6933-6942.

28 Takezawa K, Okamoto I, Okamoto W, et al. Thymidylate synthase as a determinant of pemetrexed sensitivity in non-small cell lung cancer. Br J Cancer 2011; 104: 1594-1601.

29 Wynes MW, Konopa K, Singh S, et al. Thymidylate synthase protein expression by IHC and gene copy number by SISH correlate and show great variability in non-small cell lung cancer. J Thorac Oncol 2012; 7: 982-992.

30 Simon GR, Sharma S, Cantor A, et al. ERCC1 expression is a predictor of survival in resected patients with nonsmall cell lung cancer. Chest 2005; 127: 978-983.

31 Zheng Z, Li X, Schell MJ, et al. Thymidylate synthase in situ protein expression and survival in stage I nonsmall-cell lung cancer. Cancer 2008; 112: 2765-2773.

32 Gazdar AF. DNA repair and survival in lung cancer - the two faces of Janus. N Engl J Med 2007; 356: 771-773.

33 Pierceall WE, Olaussen KA, Rousseau V, et al. Cisplatin benefit is predicted by immunohistochemical analysis of DNA repair proteins in squamous cell carcinoma but not adenocarcinoma: theranostic modeling by NSCLC constituent histological subclasses. Ann Oncol 2012; 23: 2245-2252.

34 Friboulet L, Olaussen KA, Pignon JP, et al. ERCC1 isoform expression and DNA repair in non-small-cell lung cancer. N Engl J Med 2013; 368: 1101-1110.

35 Zhang Y, Sun Y, Pan Y, et al. Frequency of driver mutations in lung adenocarcinoma from female never-smokers varies with histologic subtypes and age at diagnosis. Clin Cancer Res 2012; 18: 1947-1953.

36 Kim YT, Kim TY, Lee DS, et al. Molecular changes of epidermal growth factor receptor (EGFR) and KRAS and their impact on the clinical outcomes in surgically resected adenocarcinoma of the lung. Lung Cancer 2008; 59: $111-118$.

37 Massarelli E, Varella-Garcia M, Tang X, et al. KRAS mutation is an important predictor of resistance to therapy with epidermal growth factor receptor tyrosine kinase inhibitors in non-small-cell lung cancer. Clin Cancer Res 2007; 13: 2890-2896.

$38 \mathrm{Xu} \mathrm{J}, \mathrm{He}$ J, Yang $\mathrm{H}$, et al. Somatic mutation analysis of EGFR, KRAS, BRAF and PIK3CA in 861 patients with nonsmall cell lung cancer. Cancer Biomark 2011-2012; 10: 63-69.

39 Pesek M, Benesova L, Belsanova B, et al. Dominance of EGFR and insignificant KRAS mutations in prediction of tyrosine-kinase therapy for NSCLC patients stratified by tumor subtype and smoking status. Anticancer Res 2009; 29: 2767-2773.

40 Falchook GS, Long GV, Kurzrock R, et al. Dabrafenib in patients with melanoma, untreated brain metastases, and other solid tumours: a phase 1 dose-escalation trial. Lancet 2012; 379: 1893-1901.

41 Sen B, Peng S, Tang X, et al. Kinase-impaired BRAF mutations in lung cancer confer sensitivity to dasatinib. Sci Transl Med 2012; 4: 136ral70.

42 Paik PK, Arcila ME, Fara M, et al. Clinical characteristics of patients with lung adenocarcinomas harboring BRAF mutations. J Clin Oncol 2011; 29: 2046-2051. 
43 Marchetti A, Felicioni L, Malatesta S, et al. Clinical features and outcome of patients with non-small-cell lung cancer harboring BRAF mutations. J Clin Oncol 2011; 29: 3574-3579.

44 Kadota K, Nitadori JI, Sarkaria IS, et al. Thyroid transcription factor-1 expression is an independent predictor of recurrence and correlates with the IASLC/ATS/ERS histologic classification in patients with stage I lung adenocarcinoma. Cancer 2013; 119: 931-938.

45 Lee JG, Kim S, Shim HS. Napsin A is an independent prognostic factor in surgically resected adenocarcinoma of the lung. Lung Cancer 2012; 77: 156-161. 